Article

\title{
Assessing Spatiotemporal Characteristics of Urbanization Dynamics in Southeast Asia Using Time Series of DMSP/OLS Nighttime Light Data
}

\author{
Min Zhao ${ }^{1,2,3}$ (D), Weiming Cheng ${ }^{3,4, *}$ (D), Chenghu Zhou ${ }^{1,2,3,4}$, Manchun $\mathrm{Li}^{1,2}$, Kun Huang ${ }^{5}$ \\ and Nan Wang ${ }^{3}$ \\ 1 School of Geographic and Oceanographic Sciences, Nanjing University, Nanjing 210023, China; \\ zhaom@lreis.ac.cn (M.Z.); zhouch@lreis.ac.cn (C.Z.); limanchun@nju.edu.cn (M.L.) \\ 2 Collaborative Innovation Center of South China Sea Studies, Nanjing 210093, China \\ 3 State Key Laboratory of Resources and Environmental Information Systems, Institute of Geographic \\ Sciences and Natural Resources Research, Chinese Academy of Sciences, Beijing 100101, China; \\ wangn.14s@igsnrr.ac.cn \\ 4 Jiangsu Center for Collaborative Innovation in Geographic Information Resource Development and Application, \\ Nanjing 210023, China \\ 5 Beijing Research Center for Information Technology in Agriculture, Beijing 100097, China; huangk@nercita.org.cn \\ * Correspondence: chengwm@1reis.ac.cn; Tel.: +86-10-6488-9777
}

Received: 15 November 2017; Accepted: 26 December 2017; Published: 8 January 2018

\begin{abstract}
Intraregional spatial variations of satellite-derived anthropogenic nighttime light signals are gradually applied to identify different lighting areas with various socioeconomic activity and urbanization levels when characterizing urbanization dynamics. However, most previous partitioning approaches are carried out at local scales, easily leading to multi-standards of the extracted results from local areas, and this inevitably hinders the comparative analysis on the urbanization dynamics of the large region. Therefore, a partitioning approach considering the characteristics of nighttime light signals at both local and regional scales is necessary for studying spatiotemporal characteristics of urbanization dynamics across the large region using nighttime light imagery. Based on the quadratic relationships between the pixel-level nighttime light brightness and the corresponding spatial gradient for individual cities, we here proposed an improved partitioning approach to quickly identify different types of nighttime lighting areas for the entire region of Southeast Asia. Using the calibrated Defense Meteorological Satellite Program/Operational Line-scan System (DMSP/OLS) data with greater comparability, continuity, and intra-urban variability, the annual nighttime light imagery spanning years 1992-2013 were divided into four types of nighttime lighting areas: low, medium, high, and extremely high, associated with different intensity of anthropogenic activity. The results suggest that Southeast Asia has experienced a rapid and diverse urbanization process from 1992 to 2013. Areas with moderate or low anthropogenic activity show a faster growth rate for the spatial expansion than the developed areas with intense anthropogenic activity. Transitions between different nighttime lighting types potentially depict the trajectory of urban development, the darker areas are gradually transitioning to areas with higher lighting, indicating conspicuous trends of gradually intensified anthropogenic activity from central areas to periphery areas, and from megacities to small cities. Additionally, satellite-derived nighttime lighting areas are in good agreement with the radar-derived human settlements, with dense human settlements in extremely high and high nighttime lighting areas, while sparse human settlements in low nighttime lighting areas.
\end{abstract}

Keywords: nighttime light; urbanization; nighttime lighting types; Southeast Asia 


\section{Introduction}

Urbanization is a complicated process involving increased populations, intensified socioeconomic activity, spatially expanded urban built-up area, and infrastructures [1-3]. Measurement and monitoring of urbanization dynamics at national to global scales is significant for further understanding anthropogenic changes in the Earth's landscape and their associated environmental consequences $[4,5]$. When compared to traditional remote sensing products from visible, near-infrared or radar sensors, satellite-derived nighttime light data focus more on the intensity information of anthropogenic activity involving demographic dynamics, socioeconomic growth, and urbanization level, and therefore have been extensively used to capture more comprehensive spatiotemporal characteristics about urbanization dynamics and regional development [6-13].

Previous studies have verified that satellite-based nighttime light datasets derived from the Defense Meteorological Satellite Program's Operational Linescan System (DMSP/OLS) could provide uniform, spatially explicit, continuous, and timely observations and measurements of human activity related to settlement changes and socioeconomic dynamics [6,9,14-16]. Because of the significant positive correlations between DMSP/OLS nighttime light brightness and demographic and socioeconomic variables, plenty of studies focus on exploring the responses of nighttime light signals to regional socioeconomic activity through constructing various nighttime light indices (e.g., the total radiance of nighttime lights, the total count of lit pixels, average nighttime light intensity, etc.) $[9,15,17,18]$. Additionally, accurately and quickly identifying urban extent is also an important content for assessing urbanization dynamics using nighttime light data. Based on the spatiotemporal changes of urban extent, urbanization patterns, as well as corresponding driving factors and impacts, are also extensively discussed [19-23].

During the estimation of urbanization dynamics, the thresholding approach has been widely used to partition nighttime light imagery and thereby extract urban information at local, national, regional, and global scales. To further reduce the overestimates or underestimates of urban extent caused by the effects of over-glow and shrink of nighttime light [24,25], subsequent studies proposed various thresholding methods based on neighborhood analysis that fully consider the local spatial variations of nighttime light signals [26-28]. Su et al. [28] generated topographic maps of nighttime light signals based on spatial neighboring analysis to identify the demarcation lines between built-up areas and non-built-up areas. This method helps to accurately extract the large patches of built-up areas in urban regions and small settlements in surrounding towns, but fails to capture the spatial variations with different intensity of anthropogenic activity within the extracted results. Ma et al. [27] proposed a spatially explicit approach based on the quadratic relationship between pixel-level nighttime light brightness and corresponding spatial gradient at the local scales to identify different types of urban sub-regions experienced various development levels for more than 200 China's cities. Kamarajugedda et al. [26] delineated three urban categories for each city based on Ma's brightness gradient approach to assess urban growth dynamics for 15 major Southeast Asian cities. Though neighborhood variations of nighttime light signals were considered in these studies, these partition approaches were carried out at the city scales, easily leading to multi-standards of the extracted sub-areas from different cities, and this further hinders the comparative analysis on urbanization dynamics of the larger region. An improved approach of extracting different types of nighttime lighting areas using a unified standard is therefore needed for studying spatiotemporal characteristics of urbanization dynamics across the large region, based on nighttime light imagery.

Southeast Asian countries have experienced a rapid urbanization period since 1960, although the region's overall urbanization rate is still below the world average, the gap has been gradually narrowed [29]. Meanwhile, in the urbanization progress, huge differences exist among Southeast Asian countries, as well as among their megacities and towns [26]. A comprehensive understanding of the spatiotemporal characteristics of urbanization dynamics for Southeast Asia is essential for planning prosperous and sustainable development of the Southeast Asian region. 
The primary objective of this study is to assess the spatiotemporal characteristics of urbanization dynamics from 1992 to 2013 in Southeast Asia by analyzing the spatial changes in nighttime light brightness over time across the entire region of Southeast Asia. Firstly, the 1992-2013 DMSP/OLS nighttime light data across Southeast Asia are calibrated through intercalibration, intra-annual composition, and the enhancement of variability to obtain the more reliable time series data of DMSP/OLS nighttime light imagery for the follow-up analysis. Secondly, the pixel-level brightness gradient across the region of Southeast Asia is calculated and the annual quadratic relationships for Southeast Asia between the pixel-level nighttime light radiance and corresponding brightness gradient are established. Thirdly, based upon the fitted quadratic polynomials for each year, proper split points of the quadratic curves are set to identify the nighttime lighting areas of Southeast Asian region into four different sub-regions with various degrees of human activity, and thereby generate the long-term nighttime lighting types of Southeast Asia spanning 1992-2013. Finally, analyses on spatiotemporal characteristics of urban development from 1992 to 2013 in Southeast Asia are carried out and the responses of nighttime lighting types to high-precision human settlements are discussed.

\section{Study Area and Data Acquisition}

\subsection{Study Area}

Southeast Asia $\left(104^{\circ} 25^{\prime}-117^{\circ} 20^{\prime} \mathrm{E}, 18^{\circ} 9^{\prime}-26^{\circ} 24^{\prime}\right)$ is a sub-region of Asia with a total area of $4,470,000 \mathrm{~km}^{2}$. It is a collection of related but dissimilar states that are squeezed between the Indian Ocean and the Pacific Ocean., and contains 11 countries, including Vietnam (VN), Laos (LA), Cambodia (CAM), Thailand (TH), Myanmar (MYA), Malaysia (MAL), Singapore (SG), Indonesia (INA), Brunei (BRU), Philippines (PHI), and Timor Leste (TL) (Figure 1). This region is steadily urbanizing and the urban population of the whole region is expected to increase to 47\% by 2025 [30]. Singapore differs significantly from other countries and megacities with a current urbanization rate of $100 \%$ since 1960 [31]. The urbanization levels are different across and within countries of Southeast Asia because of diverse geography, history, and state conditions.

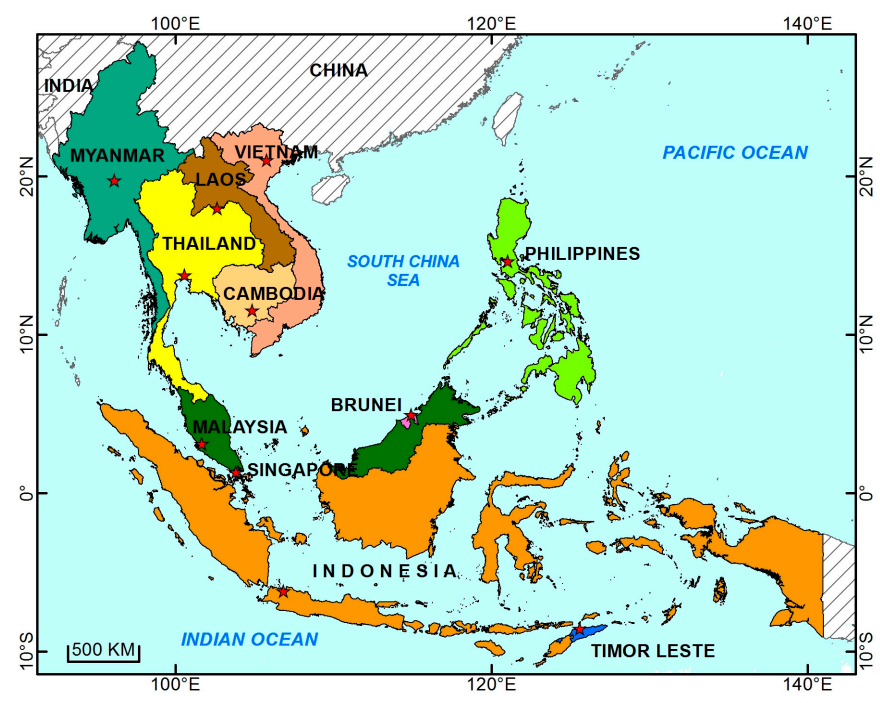

Figure 1. An overview of the Southeast Asia.

\subsection{Data Source and Preprocessing}

\subsubsection{Administrative Division Data}

The administrative boundary was derived from Global Administrative Areas website (http:/ / gadm.org/country). It provides a spatial database containing the administrative areas of all 
countries at all levels. The administrative division dataset of Southeast Asia in "shapefile" format was downloaded in this study, including boundaries of national administrative areas and corresponding first-order administrative areas in 11 Southeast Asian countries.

\subsubsection{Normal Differential Vegetation Index (NDVI) Products}

Two types of NDVI data were used in this research: the GIMMS $3 g$ NDVI data for years from 1992 to 2000, and the MOD 13A3 NDVI data for years from 2000 to 2013.

The GIMMIS $3 g$ NDVI data were obtained from NASA Earth Exchange (NEX) website (https:/ / nex.nasa.gov/nex/projects/1349/). This NDVI product is a global NDVI long-time series dataset assembled from different AVHRR sensors with a consideration of various deleterious effects (e.g., calibration loss, orbital drift, and volcanic eruptions). The latest version, 3g.v1, a 15-day product spans the period from July 1981 to December 2015, has a spatial resolution of $8 \mathrm{~km}$. In this study, the maximum value composite (MVC) method was used to choose the maximum NDVI value for each pixel per month, and then the pixel-level annual mean NDVI spanning 1992-2000 were obtained by averaging the monthly processed product.

The version 6 MODIS/Terra monthly 1-km NDVI data (1-km MOD 13A3) were obtained from Land Processes Distributed Active Archive Center (LP DAAC) website (https://lpdaacc.usgs.gov / dataset_discovery/modis/modis_products_table/mod13a3_v006). The combined NDVI product was obtained by choosing the best available pixel value with low clouds, low view angle, and the highest NDVI value from all the acquisition during the 16 day period. In this study, we generated the monthly NDVI product using the MVC method and then derived the annual mean NDVI product from 2000 to 2013. Although the NDVI data we processed cannot completely eliminate the interference from clouds and shadows, it further reduces the sensitivity of NDVI to seasonal and inter-annual fluctuations, which facilitates the follow-up analysis on estimation of urbanization dynamics.

\subsubsection{DMSP/OLS Nighttime Lights}

The version 4 composite DMSP/OLS nighttime stable light imagery, derived from National Geophysical Data Center of National Oceanic and Atmospheric Administration (NOAA/NGDC), are gridded cell-based annual cloud-free composites spanning years 1992-2013 with digital numbers (DN) ranged from 0 to 63 and a spatial resolution of 30 arc-second (approximately $1 \mathrm{~km}$ at the equator and $0.8 \mathrm{~km}$ at $\left.40^{\circ} \mathrm{N}\right)$. The 22 years DMSP nighttime imagery were separately collected by six sensors: F10 (1992-1994), F12 (1994-1999), F14 (1997-2003), F15 (2000-2007), F16 (2004-2009), and F18 (2010-2013). The images covering the region of Southeast Asia were clipped from the 34 global nighttime light images to be used as the preliminary DMSP/OLS nighttime light data in this study.

Additionally, the global radiance calibrated nighttime lights (RCNTL) for 2006 archived at the NOAA/NGDC website (https://ngdc.noaa.gov/eog/dmsp/download_radcal.html) with no sensor saturation was selected as the ideal reference imagery in this study.

\subsubsection{Integration of NDVI Time Series for Southeast Asia}

The two types of annual mean NDVI datasets for years 1992-2000 and 2000-2013 could not be directly used as auxiliary information for the calibration of DMSP data because of the sensor discrepancies. To improve the comparability, we first resampled the two NDVI datasets to a same resolution as DMSP data ( 0.00833 decimal degree under the WGS84 coordinate reference system) to ensure the spatial correspondence for pixels and then clipped the raster data for Southeast Asia. Based on the principle that the NDVI values of the chosen invariant region from GIMMS $3 g$ spanning years 1992-2000 are relative stable, a linear regression model was developed between NDVI values from GIMMS 3g in 2000 and MOD 13A3 in 2000, and was then applied to calibrate the GIMMS NDVI for years 1992-1999. The calibrated GIMMS NDVI spanning years 1992-1999 were considered to have a high comparability with MODIS NDVI spanning years 2000-2013. Based on the above integration of 
NDVI products, the time-series annual mean NDVI data spanning 1992-2013 for Southeast Asia were generated as the preliminary NDVI data in this study.

\subsubsection{Global Urban Footprint}

The global urban footprint (GUF), as derived from the Urban Thematic Exploitation Platform (Urban-TEP) (https:/ / urban-tep.eo.esa.int/\#!), are gridded cell-based images of urban settlements mask in 2012, with a spatial resolution of $12 \mathrm{~m}$. GUF are produced by the German Remote Sensing Data Center (DFD) using a newly developed method. More than 180,000 images that were derived from the German radar satellites TerraSAR X and TanDEM X spanning the years of 2010-2013, and other additional data, such as digital terrain models, are processed as the input volume, and an automated quality assurance check are conducted. Each original GUF image is presented in binary form, with 1 representing settlements and 0 representing any other land surface. The GUF data delivers unparalleled precision, which focuses on detecting characteristics of vertical structures of human habitations, and is even able to identify small villages, facilitating to the acquisition of a better understanding of the urbanization phenomenon. The 2012 GUF images of Southeast Asian region were used for the comparative analysis in this study.

\section{Methods}

\subsection{Calibration of the Nighttime Light Data}

\subsubsection{Intercalibration}

Referencing to the invariant region method proposed by Elvidge et al. [32], Liu et al. [33], and Wu et al. [34], nighttime light data of Southeast Asia from 1992 to 2013 were intercalibrated via the modified invariant region (MIR) method. Sicily was selected as the invariant region and the RCNTL for 2006 was regarded as the reference imagery. A power function regression model was used to empirically intercalibrate the annual DMSP/OLS stable nighttime light products to match the RCNTL data in 2006:

$$
D N_{c 1}=a \times D N_{c 0}^{b},
$$

where $D N_{c 1}$ is the intercalibrated $\mathrm{DN}$ value, $D N_{c 0}$ is the original $\mathrm{DN}$ value, $a$ and $b$ are coefficients.

\subsubsection{Intra-Annual Composition}

We obtained two annual composites in 1994 and from 1997 to 2007 after the intercalibration of DMSP/OLS data. To make full use of the information that was derived from two satellites for the same year and to remove the unstable lit pixels, an intra-annual composition for nighttime light data were generated by computing the average DN values of stable lit pixels, as following:

$$
D N_{c 2}=\left\{\begin{array}{c}
0, D N_{c 1}^{1}=0 \& D N_{c 1}^{2}=0 \\
\left(D N_{c 1}^{1}+D N_{c 1}^{2}\right) / 2, \text { otherwise }
\end{array},\right.
$$

where $D N_{c 2}$ is the $\mathrm{DN}$ value of the intra-annual composite, $D N_{c 1}^{1}$ and $D N_{c 1}^{2}$ are the intercalibrated DN values in the same certain year.

\subsubsection{Enhancement of Variability}

Although the RCNTL data employed during the intercalibration helped to reduce some saturation effect, a further saturation correction for the high-value pixels is still necessary when estimating the variability and heterogeneity of a region. As a pronounced inverse relationship between NDVI and DN was found in urban areas with saturated light [35], (1-NDVI) was regarded as the weight value for 
nighttime lights to reduce this saturation effect and to enhance intra-urban variability of DMSP/OLS nighttime light radiance. Thus, the output $\mathrm{DN}$ can be calculated as:

$$
D N_{c 3}=\left(1-N D V I_{c}\right) \times D N_{c 2}
$$

where $D N_{c 3}$ is the DN value of nighttime light data after NDVI calibration, $N D V I_{c}$ is the annual mean NDVI composite that is integrated by two kinds of NDVI products.

\subsection{The Spatial Gradient of Nighttime Lights}

Referencing to Ma et al.'s approach [27], we used the pixel-level brightness gradient (BG) to measure the spatial changes for the signals of artificial nighttime lighting. As illustrated in Figure 2, the brightness gradient of the central grid cell within a moving eight-cell neighborhood field is calculated as following:

$$
\begin{gathered}
G_{\text {brightness }}=\sqrt{[d D N / d x]^{2}+[d D N / d y]^{2}}, \\
{[d D N / d x]=\left[\left(D N_{3}+2 D N_{5}+D N_{8}\right)-\left(D N_{1}+2 D N_{4}+D N_{6}\right)\right] / 8,} \\
{[d D N / d y]=\left[\left(D N_{6}+2 D N_{7}+D N_{8}\right)-\left(D N_{1}+2 D N_{2}+D N_{3}\right)\right] / 8,}
\end{gathered}
$$

where $G_{\text {brightness }}$ is the spatial brightness gradient (BG) of the calibrated nighttime light data, $[d D N / d x]$ and $[d D N / d y]$ denote the rates of change of the nighttime light in the horizontal and vertical directions, $D N_{c}$ is the nighttime light brightness of the central grid cell, $D N_{1}, D N_{2}, D N_{3}, D N_{4}, D N_{5}, D N_{6}, D N_{7}$, and $D N_{8}$ are the nighttime light brightness of the eight adjacent grid cells from the central grid cell. All of the DN values are the nighttime light radiance calibrated by NDVI.

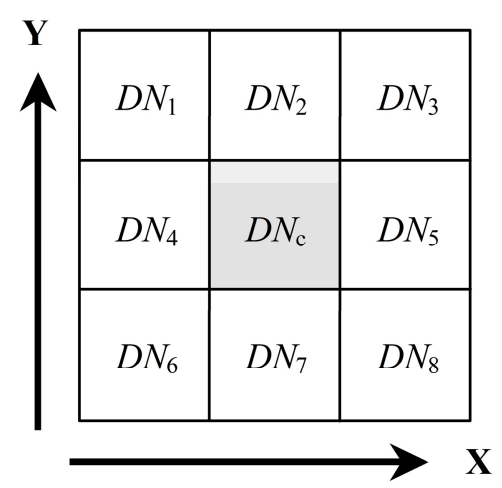

Figure 2. Schematic diagram of the calculation of the spatial brightness gradient. (Modified from Ma et al. [27]).

\subsection{Spatial Partition of Nighttime Lighting Areas}

Capturing the spatial partitions of anthropogenic nighttime lighting over time is significant in exploring the dynamics patterns of spatially expanding human settlement and economic activities. Since the quantitative relationships between the pixel-level nighttime light brightness and corresponding spatial gradient were found and used for identifying different nighttime lighting areas for cases of China and Southeast Asia [26,27], here we followed the previous studies and used a similar approach. The relationship between pixel-level nighttime light DN and corresponding BG can be estimated as following:

$$
G_{\text {brightness }}=a \times D N_{c 3}^{2}+b \times D N_{c 3}+c,
$$

where $G_{\text {brightness }}$ denotes brightness gradient; $D N_{c 3}$ denotes the $\mathrm{DN}$ value of nighttime light data after NDVI calibration; and, $a, b$, and $c$ are the coefficients of the fitted polynomial to be calculated. 
The robust quadratic relationships between the pixel-level DN and BG across Southeast Asia spanning 1992-2013 are able to identify different types of nighttime lighting areas. Ma et al. [27] partitioned nighttime light images across more than 200 of China's cities into five different types while Kamarajugedda et al. [26] divided the internal areas of 15 major Southeast Asian cities into three categories. However, the partition approaches they carried on were both at local scales (based on individual cities), easily leading to the difference and inconsistency of nighttime light characteristics for the same type in different cities. Since we focus on the spatiotemporal characteristics of different lit areas across the whole region of Southeast Asia, a unified standard for partition and comparative analysis is especially necessary. Correspondingly, the selection of split points needs to be adjusted. The approach is as following.

Based upon the diagram of the fitted quadratic polynomial (Figure 3a), the lit areas of Southeast Asian region were subdivided into four different sub-regions involving: low (nighttime light brightness range from $D N_{0}$ to $D N_{1}$, but excluding $D N_{1}$ ), medium (from $D N_{1}$ to $D N_{2}$, but excluding $D N_{2}$ ), high (from $D N_{2}$ to $D N_{3}$, but excluding $D N_{3}$ ), extremely high (from $D N_{3}$ to $D N_{4}$ ) nighttime lighting areas. As shown in Figure $3 a$, the split point $P_{2}$ is the vertex of the downward parabola with the maximum BG. $P_{0}$ and $P_{4}$ are the starting point and the ending point of the fitted parabola, respectively, where the minimum and the maximum nighttime light DN are observed. The BG value of $P_{1}$ is the average BG values of $P_{0}$ and $P_{2}$, and $P_{3}$ is the quarter-point between $P_{2}$ and $P_{4}$ in the vertical coordinate. The nighttime light pixels with DN values lower than 3 were excluded during the procession of modeling and partitioning because of the high inter-annual variability of very low DN values [26]. More detailed calculations for the DN and BG of the split points are illustrated in Table 1. The extremely high type likely represents highly developed areas with intense anthropogenic activity, the high type mainly contains developed areas with considerable anthropogenic activity, the medium type likely covers developing areas with a certain degree of anthropogenic activity, and the low type appears to be rural areas with low anthropogenic activity.
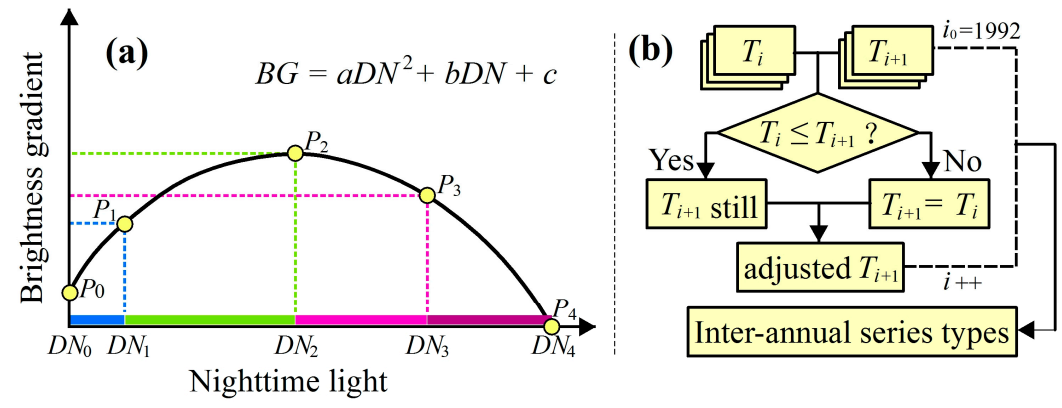

Figure 3. Schematic diagram of the (a) sub-region partitioning based on the quadratic polynomial; and (b) inter-annual series types generation.

Table 1. Calculations for the split points drawn in Figure 3a based on the quadratic polynomial.

\begin{tabular}{ccc}
\hline Point & DN Value & Brightness Gradient (BG) \\
\hline$P_{0}\left(D N_{0}, B G_{0}\right)$ & $D N_{\text {min }}$ & $f\left(D N_{\text {min }}\right)$ \\
$P_{1}\left(D N_{1}, B G_{1}\right)$ & $f^{-1}\left(B G_{1}\right), \mathrm{DN}<D N_{2}$ & $\frac{B G_{0}+B G_{2}}{2}$ \\
$P_{2}\left(D N_{2}, B G_{2}\right)$ & $-\frac{b}{2 a}$ & $\frac{4 a c-b^{2}}{4 a}$ \\
$P_{3}\left(D N_{3}, B G_{3}\right)$ & $f^{-1}\left(B G_{3}\right), \mathrm{DN}>D N_{2}$ & $\frac{3 B G_{2}+B G_{4}}{4}$ \\
$P_{4}\left(D N_{4}, B G_{4}\right)$ & $D N_{\text {max }}$ & $f\left(D N_{\text {max }}\right)$ \\
\hline
\end{tabular}

$f(D N)$ denotes the quadratic polynomial, whereas $f^{-1}(B G)$ denotes the corresponding inverse function in the specified interval.

Annually, different types of nighttime lighting areas spanning 1992-2013 of Southeast Asian region were obtained separately based on the corresponding quadratic regression formulas and the 
partition method. To reduce the inconsistencies of the inter-annual series nighttime lighting types in the Southeast Asian region, a simple inter-annual series correction was performed to correct the lighting types over time (Figure 3b). A number of studies have been conducted based upon the assumption that lit pixels that are detected in earlier nighttime light images should be maintained in later ones $[13,36]$. Therefore, we further assumed that the development levels of lit pixels identified from an earlier period should not exceed the development levels identified from a later period by considering the rapid process of urbanization in Southeast Asia. Based on this assumption, we performed the inter-annual series correction and finally derived four types of nighttime lighting areas corresponding to various development levels in Southeast Asia spanning 1992-2013.

\section{Results}

\subsection{Evaluation of the Calibration Results}

The calibration of nighttime light data contains three processes, including intercalibration, intra-annual composition, and enhancement of variability. The coefficients and fitting degree of the intercalibration model for each image are shown in Table A1. The high values of $R^{2}$ reveal the reliability of the intercalibration models. Comparisons between original nighttime light data and the calibrated results in different stages of progress are shown in Figure 4. As shown in Figure 4a,b, the discrepancies of DN values between two satellites in the same year were reduced and the abnormal fluctuations of DN values between two years from the same satellite were minimized. In general, the comparability was improved for most years in the time series (Figure 4c). Figure $4 \mathrm{~d}$ shows the time-series total radiance of nighttime light data after the enhancement of variability.
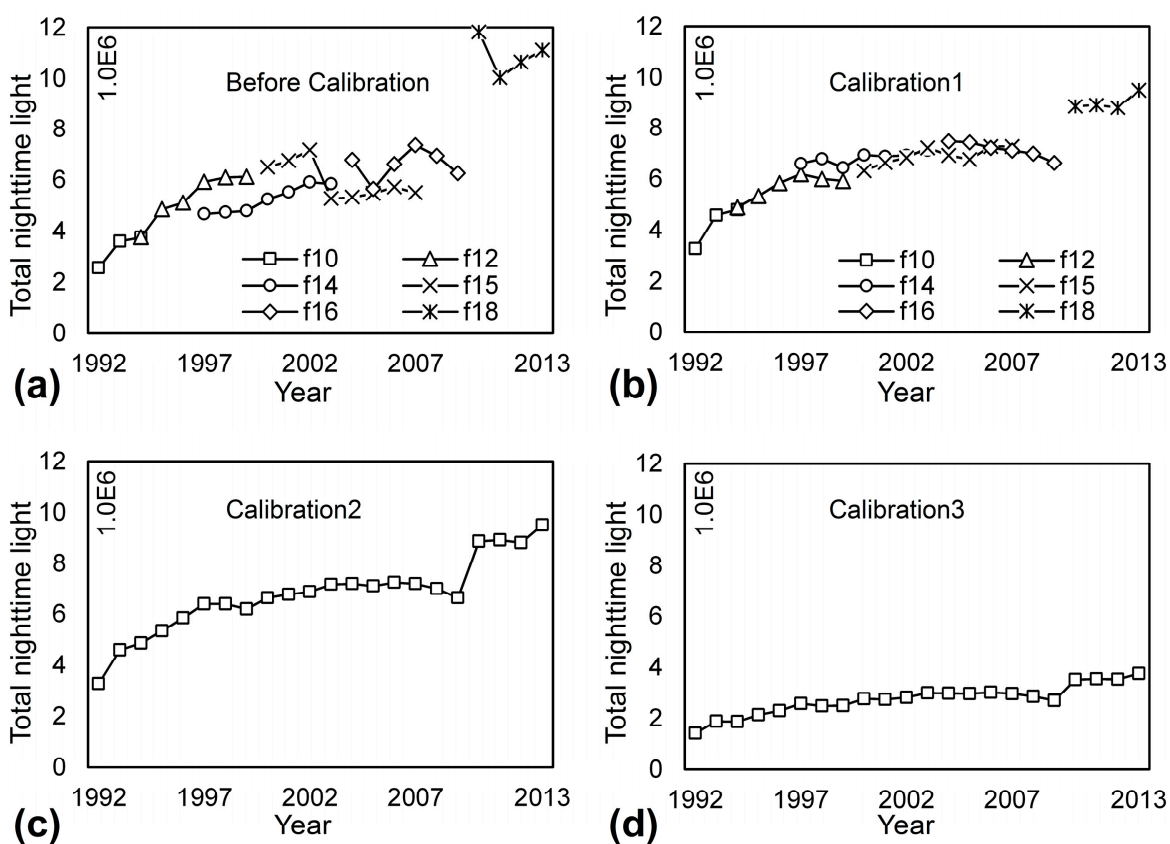

Figure 4. Comparisons of the results at different calibration stages for Southeast Asia: (a) Before calibration; (b) Calibration 1; (c) Calibration 2; (d) Calibration 3.

Figure 5 shows a spatial distribution comparison of nighttime light signals between nighttime light data before NDVI calibration (Figure 5a) and after NDVI calibration (Figure 5b) along a latitudinal transect across Bangkok and its surrounding areas. Compared with intra-annual composite nighttime lights, nighttime light images calibrated by NDVI are likely to contain more detailed information, particularly in urbanized areas, indicating a further enhancement of variability of nighttime light signals (see Figure 5c). 

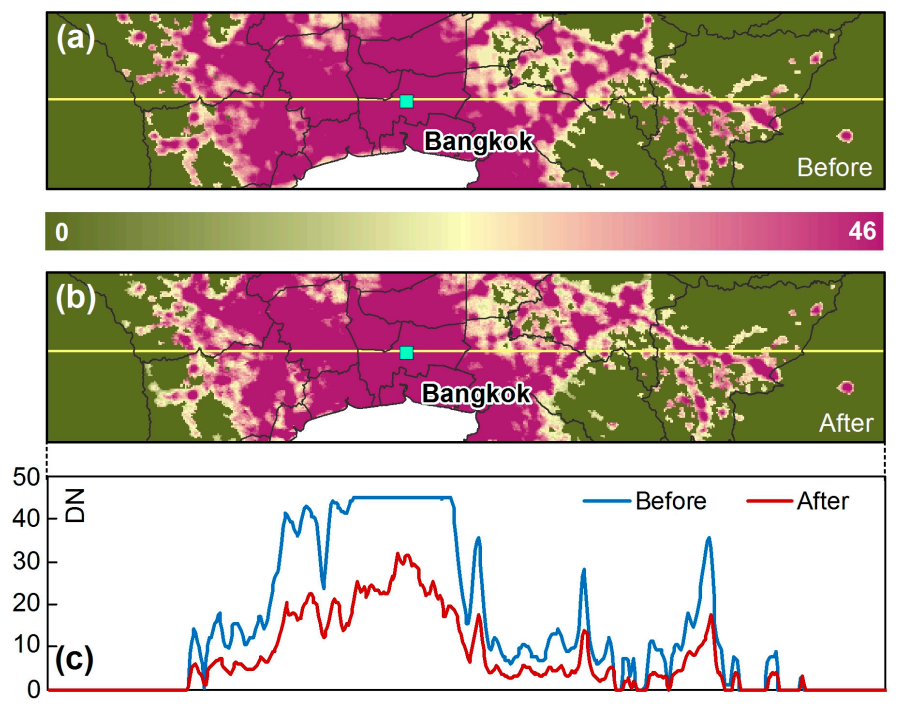

Figure 5. (a) Nighttime light before and (b) after variability enhancement over Bangkok and its surrounding areas; (c) shows profiles of pixel-level nighttime light brightness in the transect across corresponding areas.

\subsection{Spatiotemporal Trends of Nighttime Lighting Types}

Based upon the quadratic relationships that were created between pixel-level nighttime light radiance and the corresponding brightness gradient across Southeast Asia spanning years from 1992 to 2013 (Table A2), DN thresholds of each year were obtained and were then employed to extract four types of nighttime lighting areas of Southeast Asian region for these 22 years. Figure 6 reveals distinct differences in four nighttime lighting types throughout Southeast Asia during the period 1992-2013. High nighttime lighting types show a growing trend at a rate of $780.65 \mathrm{~km}^{2}$ per year, slightly more growth than extremely high nighttime lighting types, with increases of $179.94 \mathrm{~km}^{2}$ per year. An annual increase of $12,580 \mathrm{~km}^{2}$ is observed in low nighttime lighting types, conspicuously higher than medium lighting types ( $2884.2 \mathrm{~km}^{2}$ per year). Moreover, extremely high and high nighttime lighting areas experienced a combined growth of $960.59 \mathrm{~km}^{2}$ per year, which is still lower than the increasing growth trend of medium and low nighttime lighting types. These results illustrate the rapid urban growth of Southeast Asia from 1992 to 2013, and areas with moderate or low anthropogenic activity show a faster growth rate for the spatial expansion than already the developed areas with intense anthropogenic activity.

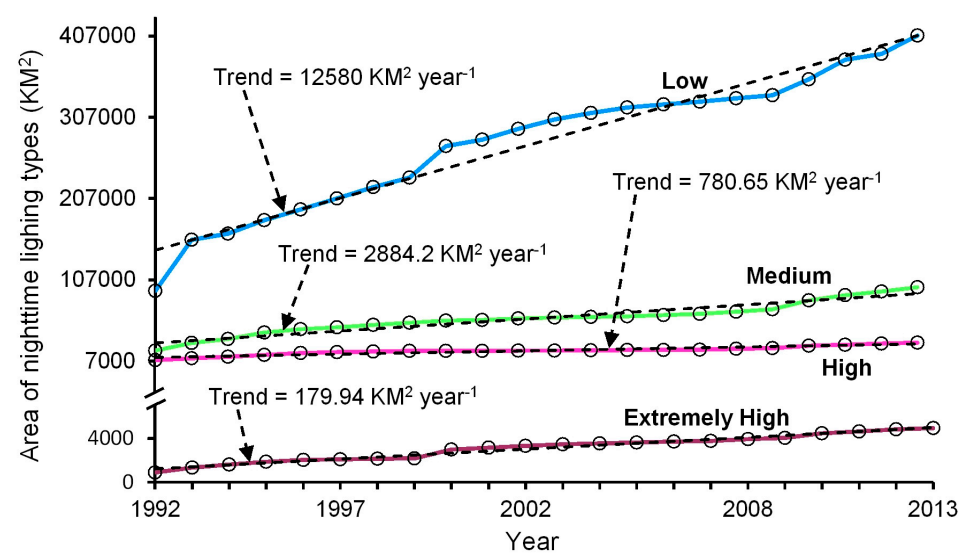

Figure 6. Changing trends in different types of nighttime lighting areas across Southeast Asia from 1992 to 2013. 
In comparison with the total growth trends of different nighttime lighting areas in Southeast Asia from 1992 to 2013, statistical results at the country scale may further portray the disparities between changes in the four nighttime lighting types. Therefore, we calculated the dynamic degrees [37] for each kind of nighttime lighting types to reflect regional differences in the change rates of four nighttime lighting types over the past decades. As shown in Figure 7, the dynamic degrees of different nighttime lighting types in 11 Southeast Asian countries and the entire region during the period from 1992 to 2013 show significant differences. Apart from Singapore, spatially expanding trends of different nighttime lighting types are observed in other countries, among which the majority of low lighting areas show evidently higher growth rate than other types. In Singapore, no low nighttime lighting areas are observed, while medium and high nighttime lighting areas appear to decline. The dynamic degrees of lower nighttime lighting areas seem to be larger than those of higher lighting areas, indicating a negative correlation between the dynamic degrees of different nighttime lighting types and the intensity of corresponding anthropogenic activity. Additionally, similar to Singapore, high and extremely high nighttime lighting areas in Brunei also exhibit higher dynamic degrees than other nighttime lighting areas. These results reveal a diverse urbanization process in Southeast Asian countries from 1992 to 2013 , in which areas with low anthropogenic activity experience a markedly spatial expansion. Moreover, developed areas still continue expanding, at a slower rate compared to developing areas.

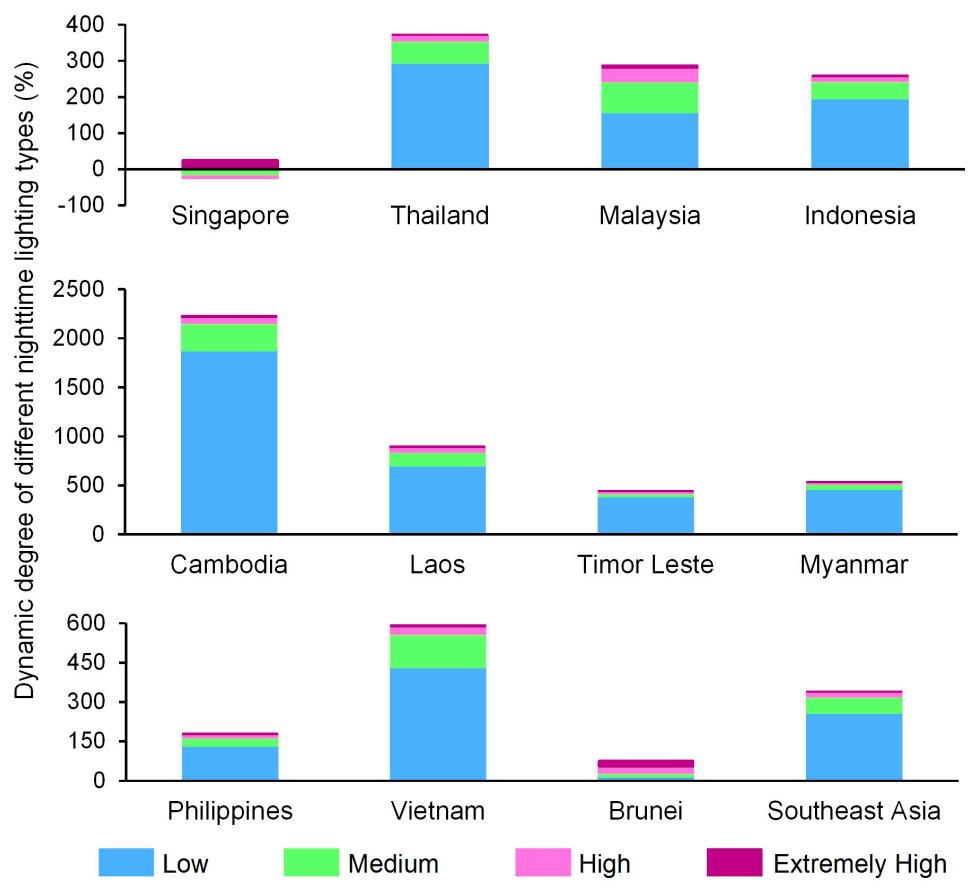

Figure 7. Dynamic degrees in different types of nighttime lighting areas across Southeast Asia between 1992 and 2013.

\subsection{Spatiotemporal Transitions of Nighttime Lighting Types}

Transitions between nocturnal lighted areas with different brightness magnitudes over time have been proved to successfully capture the dynamics of regional development [27]. The transitions of initial lit areas and areas lit after 1992 between 1992, 2002 and 2013 across Southeast Asian countries are tabulated in Tables 2 and 3, respectively. Table 2 shows that among areas that were lit after 1992, $88.99 \%$ of black background areas $(\mathrm{DN}<3)$ in 1992 were transited into low nighttime lighting areas in 2013, and $10.17 \%$ of black background areas in 1992 were developed into medium nighttime lighting region in 2013. Table 3 illustrates that among the initial lit areas, $28.91 \%$ of high and extremely high nighttime lighting areas in 2013 were sourced from low lighting areas in 1992, whereas medium nighttime lighting areas in 1992 contributed about $42.17 \%$ of the high and extremely high lighting 
areas in 2013. Comparably, among the initial lit areas, $21.81 \%$ of high and extremely high nighttime lighting areas remain unchanged over the past 22 years. More detailed information may be captured through a comprehensive comparison between Tables 2 and 3. For the high and extremely high nighttime lighting areas in 2013, 10.05\% of them came from areas lit after 1992, whereas $89.95 \%$ of them were sourced from conversions between different nighttime lighting types in the initial lit areas. Furthermore, 91.15\% of low nighttime lighting areas in 2013 came from areas lit after 1992, whereas $8.85 \%$ of the black background areas seem unchanged since 1992. For the medium nighttime lighting areas in $2013,43.43 \%$ of them were from areas lit after 1992 , whereas $56.57 \%$ of them came from the initial lit areas with low and medium nighttime light signals. These results indicate that an obviously rapid urbanization of Southeast Asian countries may likely occur in lit areas with medium nighttime light signals, and plenty of black background areas $(\mathrm{DN}<3)$ are also quickly developing into different types of nighttime lighting areas. Furthermore, the darker areas are gradually transitioning to areas with higher lighting.

Table 2. Pixel count of transition types for areas lit after 1992 across Southeast Asian countries between 1992, 2002, and 2013.

\begin{tabular}{cccccccc}
\hline \multirow{2}{*}{$\mathbf{1 9 9 2}$} & & $\mathbf{N}$ & $\mathbf{N}$ & $\mathbf{N}$ & $\mathbf{N}$ & $\mathbf{N}$ & \multirow{2}{*}{ Total } \\
\cline { 1 - 6 } $\mathbf{2 0 0 2}$ & & $\mathbf{N}$ & $\mathbf{L}$ & $\mathbf{M}$ & $\mathbf{H}$ & $\mathbf{E H}$ & \\
\hline \multirow{2}{*}{$\mathbf{2 0 1 3}$} & L & 159,092 & 212,266 & - & - & - & 371,358 \\
& M & 4796 & 25,789 & 11,868 & - & - & 42,453 \\
& H & 419 & 757 & 1404 & 698 & - & 3278 \\
& EH & 19 & 26 & 48 & 73 & 36 & 202 \\
\hline
\end{tabular}

$\mathrm{N}$ : none types (black background with DN < 3); L: low; M: medium; H: high; EH: extremely high.

Table 3. Pixel count of transition types for initial lit areas across Southeast Asian countries between 1992, 2002 and 2013.

\begin{tabular}{|c|c|c|c|c|c|c|c|c|c|c|c|c|}
\hline 1992 & & $\mathbf{L}$ & $\mathbf{L}$ & $\mathbf{L}$ & L & $\mathbf{M}$ & $\mathbf{M}$ & $\mathbf{M}$ & $\mathbf{H}$ & $\mathbf{H}$ & EH & Total \\
\hline 2002 & & $\mathbf{L}$ & $\mathbf{M}$ & $\mathbf{H}$ & EH & $\mathbf{M}$ & $\mathbf{H}$ & EH & $\mathbf{H}$ & EH & EH & \\
\hline \multirow{4}{*}{2013} & $\mathrm{~L}$ & 36,076 & - & - & - & - & - & - & - & - & - & 36,076 \\
\hline & $\mathrm{M}$ & 17,397 & 30,968 & - & - & 6926 & - & - & - & - & - & 55,291 \\
\hline & $\mathrm{H}$ & 268 & 5587 & 2637 & - & 2520 & 9487 & - & 5892 & - & - & 26,391 \\
\hline & $\mathrm{EH}$ & 4 & 143 & 232 & 133 & 51 & 514 & 564 & 519 & 1698 & 901 & 4759 \\
\hline
\end{tabular}

Intraregional spatial variations of nighttime light signals help to depict the changes of expanded human settlement and various socioeconomic activities in a region [12]. Figure 8 represents the spatial transitions of different nighttime lighting types for major cities or countries of Southeast Asia between 1992, 2002, and 2013. In Figure 8a-1, 13 study areas are partial enlarged to depict the spatial patterns of transition types at local scales, the details about these areas can be found in Table 4 . It is evident that the transitions of various types of nighttime lighting areas for diverse regions between 1992, 2002, and 2013 show pronounced spatial differences. As shown in Figure 8, in the year of 2013, Bangkok, Jakarta, Kuala Lumpur, Manila, Singapore, and Ho Chi Minh are basically covered by high and extremely high nighttime lighting areas, while Mandalay, Phnom Penh, Vientiane, Hanoi, Brunei and Yangon still contain some low and medium nighttime lighting areas near the core areas, and are also covered by considerable black background areas $(\mathrm{DN}<3)$ away from the core areas. Seen from the transition types, transition types of Jakarta, Kuala Lumpur and Singapore are mainly between the high nighttime lighting areas and extremely high nighttime lighting areas, development of these regions show a stable but highly developed trend. A certain amount of transition types between black background areas and low/medium nighttime lighting areas are found in Mandalay, Phnom Penh, Vientiane, Ho Chi Minh, Hanoi, and Yangon, these transitions basically occur far away from the core areas, where urbanization 
is or will be gradually accelerating. A great deal of the areas with nighttime light DN lower than 3 exist in Mandalay, Vientiane, Brunei, and Yangon, where regional development of the periphery areas seem a little slower. Although transitions in nighttime lighting types for different areas are different, conspicuous spatial patterns in the transition types are observed: preceding lower-level nighttime lighting areas became higher-level lit areas and their adjacent areas also developed followed by, and this kind of transition will develop into a higher level in the next stage.
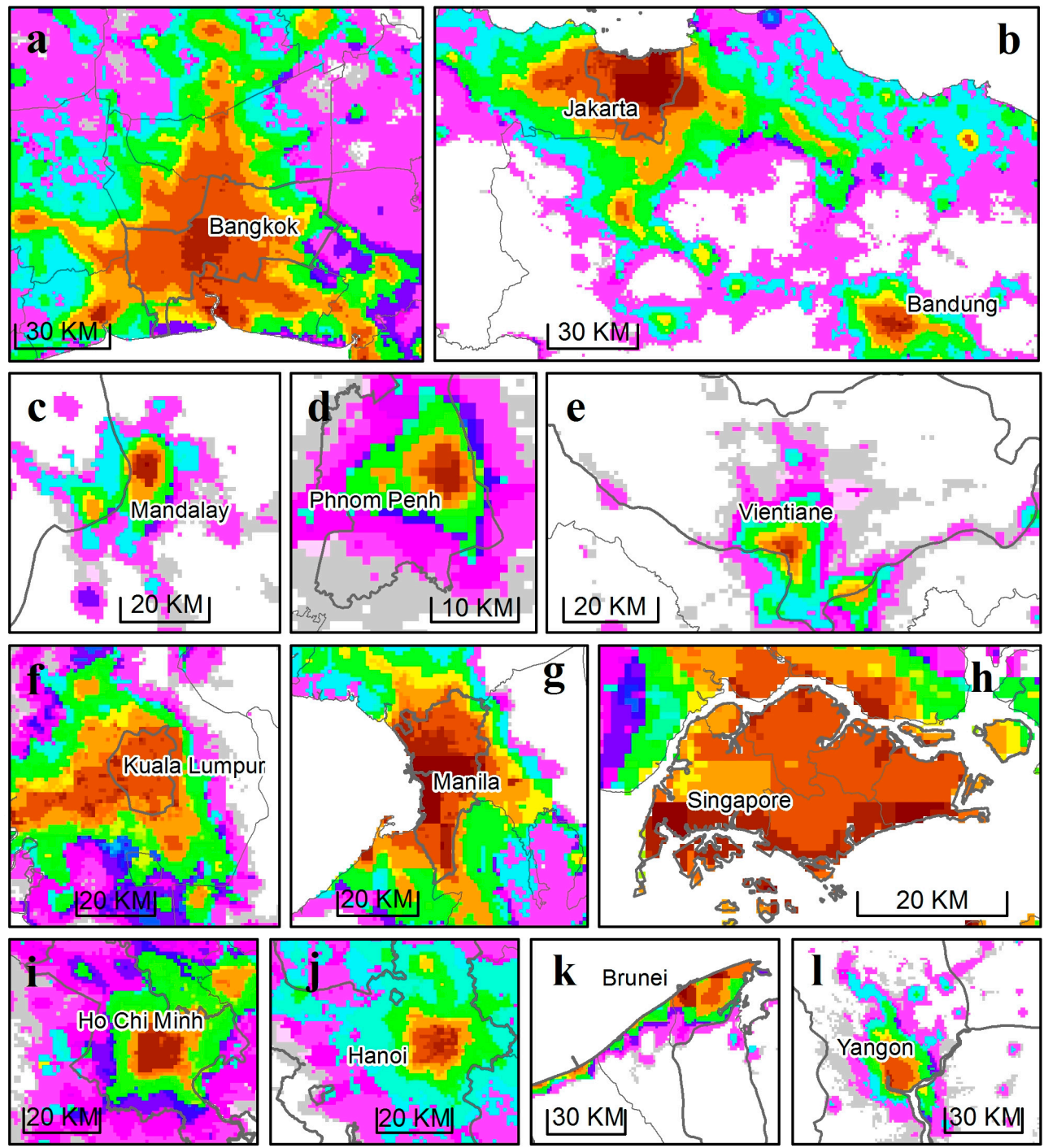

Type transitions between 1992-2002-2013. N: none; L: Low, M: Medium, H: High, EH: Extremely High

\begin{tabular}{lllllll} 
N-N-L & N-L-H & N-H-EH & L-M-M & M-M-M & H-H-H \\
\cline { 2 - 3 } N-N-M & N-L-EH & N-EH-EH & L-M-H & M-M-H & H-H-EH \\
\cline { 2 - 3 } N-N-H & N-M-M & L-L-L & L-M-EH & M-M-EH & H-EH-EH \\
\hline N-N-EH & N-M-H & L-L-M & L-H-H & M-H-H & EH-EH-EH \\
N-L-L & N-M-EH & L-L-H & L-H-EH & M-H-EH & C \\
N-L-M & N-H-H & L-L-EH & L-EH-EH & M-H-EH & Study area
\end{tabular}

Figure 8. Spatial transitions of nighttime lighting types between the year of 1992, 2002, and 2013 in several cities and countries: (a) Bangkok; (b) Jakarta; (c) Mandalay; (d) Phnom Penh; (e) Vientiane; (f) Kuala Lumpur; (g) Manila; (h) Singapore; (i) Ho Chi Minh; (j) Hanoi; (k) Brunei; and, (l) Yangon. 
Table 4. Locations and attribute information of the areas shown in Figure 8.

\begin{tabular}{cccc}
\hline Figure Code & Study Area & Country & Region Scale \\
\hline a & Bangkok & Thailand & capital; largest city \\
b & Jakarta & Indonesia & capital; largest city \\
b & Bandung & Indonesia & third-largest city \\
c & Mandalay & Myanmar & second-largest city \\
d & Phnom Penh & Cambodia & capital; largest city \\
e & Vientiane & Laos & capital \\
f & Kuala Lumpur & Malaysia & capital; largest city \\
g & Manila & Philippines & capital \\
h & Singapore & Singapore & city-state \\
i & Ho Chi Minh & Vietnam & largest city \\
j & Hanoi & Vietnam & capital; third-largest city \\
k & Brunei & Brunei & country \\
l & Yangon & Myanmar & largest city \\
\hline
\end{tabular}

\subsection{Responses of Nighttime Lighting Types to Human Settlements}

To further examine the responses of nighttime lighting types to human settlements, a comparative analysis based on GUF data were also conducted. Figure 9 shows a spatial comparison of radar-derived human settlements (GUF data) with satellite-derived nighttime lighting types of Bangkok, Ho Chi Minh, Kuala Lumpur, and Singapore in 2012. It is evident to observe a spatial correspondence of extremely high and high lighting areas with dense human settlements as well as a spatial correspondence of low nighttime lighting areas with sparse human settlements, respectively. To quantitatively evaluate the responses of nighttime lighting types to radar-derived human settlements, we computed the area percentages of the human settlements in different nighttime lighting areas for 11 Southeast Asian countries in 2012. For each Southeast Asian country, the area ratios of radar-derived human settlements in extremely high and high nighttime lighting areas are much larger than those in medium and low nighttime lighting types (seen in Figure 9). Take Indonesia as an example, $73.97 \%$ of the extremely high nighttime lighting areas of this country belong to human settlements, whereas $6.923 \%$ of the low nighttime lighting areas are human settlements. When considering that the spatial resolution of these two datasets is different, and that human settlements are not the only characterization of the strong intensity of anthropogenic activity, our results for the area percentages are reasonable. More details about the comparisons between extracted nighttime lighting types and radar-derived human settlements can be seen in Figure 10. Because of the high spatial resolution of GUF data $(12 \mathrm{~m})$, human settlements located in lit areas with extremely high and high nighttime lights can be regarded as the urban construction land, and human settlements located in lit areas with medium nighttime lights may be suburban construction land, and human settlements that are located in low nighttime lighting types are likely to be small villages. 


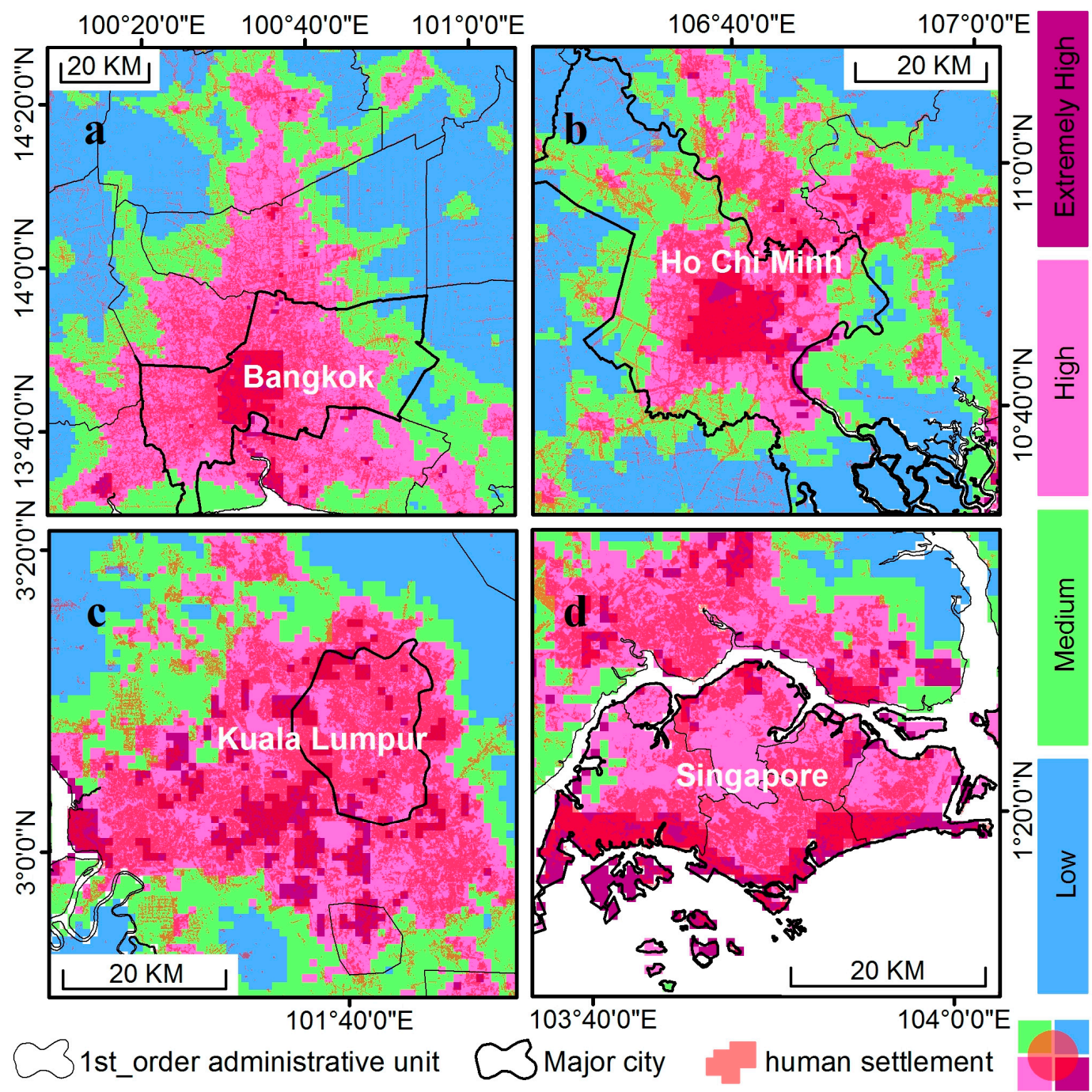

Figure 9. Comparisons of nighttime light imagery-derived sub-regions with radar-derived human settlements in 2012 over several cities and countries: (a) Bangkok; (b) Ho Chi Minh; (c) Kuala Lumpur; and, (d) Singapore.

\begin{tabular}{c|c|c|c|c|c|c|c|c|c|c|c|}
\hline L & 7.492 & 2.827 & 4.214 & 2.826 & 4.253 & 3.709 & NONE & 6.923 & 1.880 & 5.608 & 2.755 \\
\hline M & 15.76 & 10.62 & 13.28 & 8.602 & 18.22 & 13.48 & 0.863 & 18.63 & 7.270 & 18.04 & 33.49 \\
\hline H & 40.72 & 33.92 & 46.18 & 33.92 & 45.10 & 43.64 & 40.97 & 50.91 & 23.68 & 50.16 & 75.99 \\
\hline EH & 62.13 & 49.64 & 64.88 & 59.29 & 67.37 & 50.83 & 51.84 & 73.97 & 19.65 & 78.18 & 26.76 \\
\hline & VN & LA & CAM & TH & MYA & MAL & SG & INA & BRU & PHI & TL \\
\hline
\end{tabular}

Figure 10. Area percentages (\%) of radar-derived human settlements of 2012 in different nighttime lighting areas for 11 Southeast Asian countries. 


\section{Discussion}

\subsection{Comparison with Existing Research}

In this study, we improved the BG partitioning approach of DMSP/OLS nighttime light imagery proposed by previous studies to quickly identify different types of nighttime lighting areas across Southeast Asia over the past 22 years. When compared with related research by Kamarajugedda et al. [26] and Ma et al. [27] concerning 274 China's cities and 15 major Southeast Asian cities, respectively, the partitioning approach that is carried out in this study has two main differences: the scale of the fitted quadratic polynomial; and, the calculations of partitioning intervals.

\subsubsection{The Scale of the Fitted Quadratic Polynomial}

Unlike previous studies, which performed quadratic regressions for pixel-level DN and BG at the city scales, we established the quadratic regressions at the scale of the entire Southeast Asian region in this study. Our considerations are mainly based on three aspects.

The first consideration is using unified criteria to characterize urbanization stages. Because our focus is on the overall urbanization characteristics of Southeast Asia, it may lead to the multiple criteria for the identification of the same type of nighttime lighting areas in different cities if the quadratic relationships between pixel-level nighttime light brightness and corresponding spatial gradient are established at the city scales. Thus, identifying different lighting types with a unified standard may reduce the differences and the inconsistency of nighttime light characteristics for the same nighttime lighting type in different regions, and this is helpful to further compare and analyze the overall trend and local difference of urbanization across Southeast Asia.

The second consideration is assessing the urbanization dynamics of the entire Southeast Asia region to avoid that nighttime lighting types in some areas cannot be identified caused by the insignificance of regression results at local scales. Actually, the administrative units of Southeast Asian countries are generally much smaller than the same level of administrative units in China. For instance, sizes of the first-order administrative units (mainly refers to provinces) in Vietnam, Laos, Thailand, Cambodia, Philippines, Timor Leste, Singapore, and Brunei are similar to China's second-order administrative units (mainly refers to cities). Additionally, there exist huge disparities of the socioeconomic and urbanization characteristics in central and peripheral cities of Southeast Asian countries. The small cities that are far away from megacities in Southeast Asia always have low nighttime light emissions in their urban core areas, and therefore the quadratic fittings of most small cities between pixel-level nighttime light brightness and corresponding brightness gradient are probably insignificant. A similar situation is also applied to highly developed megacities, whose nighttime light brightness in periphery areas are generally high. The particularities of nighttime light brightness in periphery cities and highly-developed megacities are easily lead to insignificant quadratic relationships between nighttime light radiance and the corresponding brightness gradient at the city scales, and thereby hamper the partitioning of nighttime lighting types across the entire region of Southeast Asia.

The third consideration is optimizing the partition approach to quickly identify different types of nighttime lighting areas for the entire Southeast Asia region. It is a daunting task to separately establish fitting relationships, calculate partitioning intervals, and extract and identify different nighttime lighting types for more than 2000 cities across Southeast Asia from 1992 to 2013. Therefore, an improved partitioning approach of nighttime light imagery is of great significance for quickly identifying urbanization dynamics of large region under long time series.

Based upon the above considerations, we performed quadratic regressions between pixel-level DN and BG at the scale of Southeast Asia to identify different nighttime lighting types for the entire region with a unified classification standard. Although our approach may ignore some details of non-common characteristics of urbanization, it has little impact on the overall analysis of urbanization 
dynamics for Southeast Asia, and our partitioning approach may help to provide a reference on the identification of different nighttime lighting types for large region.

\subsubsection{The Calculations of Partitioning Intervals}

In previous related studies, Ma et al. [27] focused on more than 200 cities and divided nighttime light images of each city into five types of nighttime lighting areas (low, medium-low, medium, medium-high, and high) with different intensity of anthropogenic activity, whereas Kamarajugedda et al. [26] took 15 major cities of Southeast Asia as the study area and extracted three categories (countryside, peri-urban, and core-urban) for each city. According to their research objectives, the calculations of partitioning intervals for these two studies are also different. In this study, in order to reduce the fragmentation of nighttime lit areas, as well as to reflect more details of urbanization, we improved previous classifications and divided nighttime light image across Southeast Asia into four types, involving low, medium, high, and extremely high nighttime lighting areas. Correspondingly, the selection of split points needs to be adjusted.

When considering that the brightness gradients in small cities and highly developed megacities are relatively lower, potentially closer to the $\mathrm{X}$-axis in the scatter plot between nighttime light brightness and corresponding gradient, the fitting results are likely to exhibit a form of a flat fitted parabola. To reduce the possibility of mistakenly identifying higher nighttime lighting areas as lower nighttime lighting areas, we moved the split points of the fitted quadratic curve a bit to the left when compared to previous studies, these points were used to identify medium, high, and extremely high nighttime lighting types, more calculation details can be seen in the analyses on split points.

In summary, the improved partitioning approach is proposed based on the comprehensive consideration of the study objective and the Southeast Asian state conditions. The practical advantage of this improved method in our study is that it achieves a unified identification for different districts of Southeast Asia that are associated with various levels of urban development, when considering both local variation and overall consistency of nighttime light signals, which provides a helpful attempt to quickly realize the large-scale and long-term monitoring of urbanization dynamics using nighttime light images.

\subsection{Limitation and Future Perspective}

Recently composites of nighttime light imagery derived from the Visible Infrared Imaging Radiometer Suite (VIIRS) instrument aboard the Suomi National Polar orbiting Partnership satellite (Suomi-NPP) have higher spatial resolution, lower detection limit and less saturation effects than DMSP data, enable the detection of clearer and more detailed information from anthropogenic light emissions [38,39]. As the new generation of nighttime light data, VIIRS data demonstrates significant advantages over DMSP imagery, and is available since 2012. Integrating these two datasets to produce a time series of continuous and consistently monitored data from 1992 to the present day has potential for characterizing the spatiotemporal patterns of socioeconomic activity and urbanization dynamics, and related work is being conducted by Zhu et al. [40]. Although the VIIRS data and the integrated data generated from VIIRS data and DMSP data differ from the DMSP data in terms of the spatial resolution and light detection capability, the variations of brightness gradient from urban core areas to rural areas are similar. Thus, different forms of quadratic polynomial relationships between nighttime light radiance and corresponding gradient still exist in VIIRS nighttime light images. Further studies are needed to investigate the most appropriate matching patterns between the split points and fitting scales of the partition method by using the extended temporal coverage dataset in order to realize the long-term continuous monitoring of spatiotemporal characteristics of regional development.

\section{Conclusions}

Time series variations of DMSP/OLS stable nighttime light signals, which are closely correlated with demographic and socioeconomic dynamics, provide an efficient way to track the spatiotemporal 
characteristics of urbanization. However, the raw data from version 4 DMSP/OLS stable nighttime lights time series dataset cannot be used directly for temporal analysis due to the absence of on-board calibration and intercalibration, as well as the saturation effects that exist in the urban central areas. To improve the comparability and continuity, and to enhance urban variability of the nighttime light signals, we calibrated the nighttime light images by three steps, including intercalibration, intra-annual composition, and enhancement of variability to systematically generate calibrated time series nighttime light data for assessing dynamics of regional development across Southeast Asia during the period of 1992-2013. In order to make full advantage of the spatial variations of nighttime light signals and to assess the spatiotemporal dynamics of urbanization across the entire Southeast Asia using a unified standard, we calculated the pixel-level brightness gradient of Southeast Asia that was proposed by previous studies and established the yearly quadratic relationships between the pixel-level nighttime light radiance and corresponding brightness gradient at the scale of the entire region. Based on comprehensive consideration, appropriate split points were set to quantitatively partition the yearly DMSP/OLS imagery across Southeast Asia into four sub-regions: low, medium, high, and extremely high nighttime lighting areas, which were potentially associated with various degrees of demographic and socioeconomic activity. Through analyses on spatiotemporal trends and transitions of different types of nighttime lighting areas, as well as the responses of different nighttime lighting types to human settlements, the spatiotemporal characteristics of urbanization dynamics across Southeast Asia from 1992 to 2013 were finally captured.

Generally, Southeast Asia has experienced a rapid and diverse urbanization process, spanning the years 1992-2013. When compared to high and extremely high nighttime lighting areas with intense anthropogenic activity, low nighttime lighting areas seem to exhibit a more obvious spatial expansion. Spatiotemporal transitions between different nighttime lighting types potentially depict the urbanization trajectory, which indicates conspicuous trends of gradually intensified anthropogenic activity from central areas to periphery areas, and from megacities to small cities. In addition, responses of nighttime lighting types to human settlements further delineate the dynamics of regional development. The satellite derived nighttime lighting areas are in good agreement with the radar derived human settlements with a spatial correspondence of extremely high and high lighting areas with dense human settlements, as well as a spatial correspondence of low nighttime lighting areas with sparse human settlements.

Acknowledgments: The authors express gratitude to anonymous reviewers for improving the manuscript. This study was supported by the NSFC Key Program (No. 41590845), the NSFC Innovation Research Group Project (No. 41421001), and the Major State Basic Research Development Program of China (No. 2015CB954101).

Author Contributions: Min Zhao, Weiming Cheng and Chenghu Zhou conceived and designed the experiments; Min Zhao and Kun Huang carried out the method; Zhao Min performed the analysis and wrote the paper; Manchun Li, Chenghu Zhou and Nan Wang reviewed and edited the manuscript.

Conflicts of Interest: The authors declare no conflict of interest.

\section{Appendix A}

Table A1. Intercalibration model coefficients of each image.

\begin{tabular}{cccccc}
\hline No. & Satellite & Year & $\mathbf{a}$ & $\mathbf{b}$ & $\mathbf{R}^{\mathbf{2}}$ \\
\hline 1 & F10 & 1992 & 1.0390 & 1.074 & 0.9923 \\
2 & F10 & 1993 & 1.5700 & 0.919 & 0.9910 \\
3 & F10 & 1994 & 1.5900 & 0.9155 & 0.9932 \\
4 & F12 & 1994 & 1.0770 & 0.9859 & 0.9917 \\
5 & F12 & 1995 & 1.3480 & 0.9239 & 0.9937 \\
6 & F12 & 1996 & 1.4530 & 0.9100 & 0.9933 \\
7 & F12 & 1997 & 1.2140 & 0.9444 & 0.9941 \\
8 & F12 & 1998 & 1.2610 & 0.9084 & 0.9944 \\
\hline
\end{tabular}


Table A1. Cont.

\begin{tabular}{cccccc}
\hline No. & Satellite & Year & $\mathbf{a}$ & $\mathbf{b}$ & $\mathbf{R}^{\mathbf{2}}$ \\
\hline 9 & F12 & 1999 & 1.2500 & 0.9059 & 0.9933 \\
10 & F14 & 1997 & 1.4820 & 0.9817 & 0.9930 \\
11 & F14 & 1998 & 1.9310 & 0.8785 & 0.9929 \\
12 & F14 & 1999 & 1.6640 & 0.9147 & 0.9959 \\
13 & F14 & 2000 & 1.8760 & 0.8574 & 0.9960 \\
14 & F14 & 2001 & 1.4650 & 0.9363 & 0.9966 \\
15 & F14 & 2002 & 1.7610 & 0.8383 & 0.9942 \\
16 & F14 & 2003 & 1.6280 & 0.889 & 0.9970 \\
17 & F15 & 2000 & 1.4320 & 0.8506 & 0.9923 \\
18 & F15 & 2001 & 1.1610 & 0.9374 & 0.9937 \\
19 & F15 & 2002 & 1.0980 & 0.9467 & 0.9948 \\
20 & F15 & 2003 & 1.8230 & 0.8815 & 0.9944 \\
21 & F15 & 2004 & 1.6450 & 0.9044 & 0.9969 \\
22 & F15 & 2005 & 1.7500 & 0.8586 & 0.9944 \\
23 & F15 & 2006 & 1.6580 & 0.8938 & 0.9977 \\
24 & F15 & 2007 & 1.7850 & 0.8824 & 0.9979 \\
25 & F16 & 2004 & 1.4090 & 0.9044 & 0.9932 \\
26 & F16 & 2005 & 1.3890 & 0.9793 & 0.9977 \\
27 & F16 & 2006 & 1.1420 & 0.9827 & 0.9990 \\
28 & F16 & 2007 & 1.0810 & 0.9588 & 0.9968 \\
29 & F16 & 2008 & 1.2040 & 0.9348 & 0.9951 \\
30 & F16 & 2009 & 1.3200 & 0.9228 & 0.9949 \\
31 & F18 & 2010 & 0.8010 & 0.9771 & 0.9963 \\
32 & F18 & 2011 & 1.4390 & 0.8205 & 0.9916 \\
33 & F18 & 2012 & 1.0190 & 0.9285 & 0.9963 \\
34 & F18 & 2013 & 1.2810 & 0.8603 & 0.9950 \\
\hline
\end{tabular}

Table A2. Coefficients of the quadratic polynomials for partitioning images from 1992 to 2013.

\begin{tabular}{ccccc}
\hline Year & $\mathbf{a}$ & $\mathbf{b}$ & $\mathbf{c}$ & $\mathbf{R}^{\mathbf{2}}$ \\
\hline 1992 & -0.006272 & 0.3581 & -0.1520 & 0.7540 \\
1993 & -0.007492 & 0.3520 & -0.4060 & 0.7392 \\
1994 & -0.008074 & 0.3603 & -0.4186 & 0.7452 \\
1995 & -0.008351 & 0.3360 & -0.3476 & 0.7201 \\
1996 & -0.00874 & 0.3517 & -0.3926 & 0.7252 \\
1997 & -0.007868 & 0.3660 & -0.4985 & 0.7857 \\
1998 & -0.008155 & 0.3405 & -0.4484 & 0.7559 \\
1999 & -0.008209 & 0.3399 & -0.4743 & 0.7474 \\
2000 & -0.006048 & 0.3080 & -0.3878 & 0.7477 \\
2001 & -0.006207 & 0.3402 & -0.4097 & 0.7753 \\
2002 & -0.006679 & 0.3280 & -0.4120 & 0.7427 \\
2003 & -0.005403 & 0.3228 & -0.3689 & 0.7866 \\
2004 & -0.006221 & 0.3455 & -0.4316 & 0.7677 \\
2005 & -0.005472 & 0.3345 & -0.3934 & 0.7934 \\
2006 & -0.005931 & 0.3402 & -0.4111 & 0.7796 \\
2007 & -0.006236 & 0.3334 & -0.3334 & 0.7434 \\
2008 & -0.006794 & 0.3364 & -0.3134 & 0.6917 \\
2009 & -0.007111 & 0.3583 & -0.3674 & 0.7009 \\
2010 & -0.00811 & 0.3171 & -0.2354 & 0.6810 \\
2011 & -0.008429 & 0.3222 & -0.3227 & 0.7444 \\
2012 & -0.007755 & 0.3185 & -0.1935 & 0.7419 \\
2013 & -0.007508 & 0.2991 & -0.1245 & 0.6972 \\
\hline
\end{tabular}




\section{References}

1. Cohen, B. Urbanization in developing countries: Current trends, future projections, and key challenges for sustainability. Technol. Soc. 2006, 28, 63-80. [CrossRef]

2. Montgomery, M.R. The urban transformation of the developing world. Science 2008, 319, 761-764. [CrossRef] [PubMed]

3. Zhao, M.; Cheng, W.M.; Zhou, C.H.; Li, M.C.; Wang, N.; Liu, Q.Y. GDP spatialization and economic differences in South China based on NPP-VIIRS nighttime light imagery. Remote Sens. 2017, 9, 673. [CrossRef]

4. Johnson, M.P. Environmental impacts of urban sprawl: A survey of the literature and proposed research agenda. Environ. Plan. A 2001,33, 717-735. [CrossRef]

5. Shukla, V.; Parikh, K. The environmental consequences of urban growth: Cross-national perspectives on economic development, air pollution, and city size. Urban Geogr. 1992, 13, 422-449. [CrossRef]

6. Elvidge, C.D.; Baugh, K.E.; Anderson, S.J.; Sutton, P.C.; Ghosh, T. The night light development index (NLDI): A spatially explicit measure of human development from satellite data. Soc. Geogr. Discuss. 2012, 7, $23-35$. [CrossRef]

7. $\quad$ Elvidge, C.D.; Baugh, K.E.; Dietz, J.B.; Bland, T.; Sutton, P.C.; Kroehl, H.W. Radiance calibration of DMSP-OLS low-light imaging data of human settlements. Remote Sens. Environ. 1999, 68, 77-88. [CrossRef]

8. Ghosh, T.; Anderson, S.J.; Elvidge, C.D.; Sutton, P.C. Using nighttime satellite imagery as a proxy measure of human well-being. Sustainability 2013, 5, 4988-5019. [CrossRef]

9. Ghosh, T.; Powell, R.L.; Elvidge, C.D.; Baugh, K.E.; Sutton, P.C.; Anderson, S. Shedding light on the global distribution of economic activity. Open Geogr. 2010, 3, 148-161. [CrossRef]

10. Sutton, P.C. A scale-adjusted measure of "Urban sprawl" using nighttime satellite imagery. Remote Sens. Environ. 2003, 86, 353-369. [CrossRef]

11. Sutton, P.C.; Roberts, D.; Elvidge, C.; Baugh, K. Census from heaven: An estimate of the global human population using night-time satellite imagery. Int. J. Remote Sens. 2001, 22, 3061-3076. [CrossRef]

12. Zhao, M.; Cheng, W.M.; Zhou, C.H.; Li, M.C.; Wang, N.; Liu, Q.Y. Spatial differentiation and morphologic characteristics of China's urban core zones based on geomorphologic partition. J. Appl. Remote Sens. 2017, 11, 016041. [CrossRef]

13. Zhao, M.; Cheng, W.M.; Liu, Q.Y.; Wang, N. Spatiotemporal measurement of urbanization levels based on multiscale units: A case study of the Bohai Rim Region in China. J. Geogr. Sci. 2016, 26, 531-548. [CrossRef]

14. Chen, X.; Nordhaus, W.D. Using luminosity data as a proxy for economic statistics. Proc. Natl. Acad. Sci. USA 2011, 108, 8589-8594. [CrossRef] [PubMed]

15. Doll, C.N.H.; Muller, J.P.; Morley, J.G. Mapping regional economic activity from night-time light satellite imagery. Ecol. Econ. 2006, 57, 75-92. [CrossRef]

16. Ma, T.; Zhou, C.H.; Pei, T.; Haynie, S.; Fan, J.F. Quantitative estimation of urbanization dynamics using time series of DMSP/OLS nighttime light data: A comparative case study from China's cities. Remote Sens. Environ. 2012, 124, 99-107. [CrossRef]

17. Zhang, Q.L.; Seto, K.C. Mapping urbanization dynamics at regional and global scales using multi-temporal DMSP/OLS nighttime light data. Remote Sens. Environ. 2011, 115, 2320-2329. [CrossRef]

18. Zhou, Y.K.; Ma, T.; Zhou, C.H.; Xu, T. Nighttime light derived assessment of regional inequality of socioeconomic development in China. Remote Sens. 2015, 7, 1242-1262. [CrossRef]

19. Cao, X.; Chen, J.; Imura, H.; Higashi, O. A SVM-based method to extract urban areas from DMSP-OLS and SPOT VGT data. Remote Sens. Environ. 2009, 113, 2205-2209. [CrossRef]

20. Henderson, M.; Yeh, E.T.; Gong, P.; Elvidge, C.; Baugh, K. Validation of urban boundaries derived from global night-time satellite imagery. Int. J. Remote Sens. 2003, 24, 595-609. [CrossRef]

21. Xiao, P.F.; Wang, X.H.; Feng, X.Z.; Zhang, X.L.; Yang, Y.K. Detecting China's urban expansion over the past three decades using nighttime light data. IEEE J. Sel. Top. Appl. Earth Obs. Remote Sens. 2014, 7, 4095-4106. [CrossRef]

22. Xie, Y.H.; Weng, Q.H. Updating urban extents with nighttime light imagery by using an object-based thresholding method. Remote Sens. Environ. 2016, 187, 1-13. [CrossRef]

23. Zhou, Y.Y.; Smith, S.J.; Elvidge, C.D.; Zhao, K.G.; Thomson, A.; Imhoff, M. A cluster-based method to map urban area from DMSP/OLS nightlights. Remote Sens Environ. 2014, 147, 173-185. [CrossRef] 
24. Elvidge, C.D.; Baugh, K.E.; Kihn, E.A.; Kroehl, H.W.; Davis, E.R. Mapping city lights with nighttime data from the DMSP operational linescan system. Photogramm. Eng. Remote Sens. 1997, 63, 727-734.

25. Imhoff, M.L.; Lawrence, W.T.; Stutzer, D.C.; Elvidge, C.D. A technique for using composite DMSP-OLS “City Lights" satellite data to map urban area. Remote Sens. Environ. 1997, 61, 361-370. [CrossRef]

26. Kamarajugedda, S.A.; Mandapaka, P.V.; Lo, E.Y.M. Assessing urban growth dynamics of major Southeast Asian cities using night-time light data. Int. J. Remote Sens. 2017, 38, 6073-6093. [CrossRef]

27. Ma, T.; Zhou, Y.K.; Zhou, C.H.; Haynie, S.; Pei, T.; Xu, T. Night-time light derived estimation of spatio-temporal characteristics of urbanization dynamics using DMSP/OLS satellite data. Remote Sens. Environ. 2015, 158, 453-464. [CrossRef]

28. Su, Y.X.; Chen, X.Z.; Wang, C.Y.; Zhang, H.O.; Liao, J.S.; Ye, Y.Y.; Wang, C.J. A new method for extracting built-up urban areas using DMSP-OLS nighttime stable lights: A case study in the Pearl River Delta, southern China. GISci. Remote Sens. 2015, 52, 218-238. [CrossRef]

29. Wang, Y.; Zhang, H.O.; Huang, G.Z.; Jin, L.X.; Lin, L.Y. Different characteristics and driving factors of the comprehensive urbanization levels of countries in Southeast Asia. Trop. Geogr. 2015, 35, 680-686. (In Chinese) [CrossRef]

30. Aritenang, A.F. Urbanization in Southeast Asia: Issues and impacts. Bull. Indones. Econ. Stud. 2014, 50, 144. [CrossRef]

31. Li, X.H.; Liu, J.L.; Gibson, V.; Zhu, Y.G. Urban sustainability and human health in China, East Asia and Southeast Asia. Curr. Opin. Environ. Sustain. 2012, 4, 436-442. [CrossRef]

32. Elvidge, C.D.; Ziskin, D.; Baugh, K.E.; Tuttle, B.T.; Ghosh, T.; Pack, D.W.; Erwin, E.H.; Zhizhin, M. A fifteen year record of global natural gas flaring derived from satellite data. Energies 2009, 2, 595-622. [CrossRef]

33. Liu, Z.F.; He, C.Y.; Zhang, Q.F.; Huang, Q.X.; Yang, Y. Extracting the dynamics of urban expansion in China using DMSP-OLS nighttime light data from 1992 to 2008. Landsc. Urban Plan. 2012, 106, 62-72. [CrossRef]

34. Wu, J.S.; He, S.B.; Peng, J.; Li, W.F.; Zhong, X.H. Intercalibration of DMSP-OLS night-time light data by the invariant region method. Int. J. Remote Sens. 2013, 34, 7356-7368. [CrossRef]

35. Zhang, Q.L.; Schaaf, C.; Seto, K.C. The vegetation adjusted NTL urban index: A new approach to reduce saturation and increase variation in nighttime luminosity. Remote Sens. Environ. 2013, 129, 32-41. [CrossRef]

36. He, C.Y.; Shi, P.J.; Li, J.G.; Chen, J.; Pan, Y.Z.; Li, J.; Zhuo, L.; Ichinose, T. Restoring urbanization process in China in the 1990s by using non-radiance-calibrated DMSP/OLS nighttime light imagery and statistical data. Chin. Sci. Bull. 2006, 51, 1614-1620. [CrossRef]

37. Liu, J.Y.; Kuang, W.H.; Zhang, Z.X.; Xu, X.L.; Qin, Y.W.; Ning, J.; Zhou, W.; Zhang, S.; LI, R.; Yan, C.; et al. Spatiotemporal characteristics, patterns, and causes of land-use changes in China since the late 1980s. J. Geogr. Sci. 2014, 24, 195-210. [CrossRef]

38. Baugh, K.; Hsu, F.C.; Elvidge, C.D.; Zhizhin, M. Nighttime lights compositing using the VIIRS day-night band: Preliminary results. Proc. Asia Pac. Adv. Netw. 2013, 35, 70-86. [CrossRef]

39. Miller, S.D.; Mills, S.P.; Elvidge, C.D.; Lindsey, D.T.; Lee, T.F.; Hawkins, J.D. Suomi satellite brings to light a unique frontier of nighttime environmental sensing capabilities. Proc. Natl. Acad. Sci. USA 2012, 109, 15706-15711. [CrossRef] [PubMed]

40. Zhu, X.B.; Ma, M.G.; Yang, H.; Ge, W. Modeling the spatiotemporal dynamics of gross domestic product in China using extended temporal coverage nighttime light data. Remote Sens. 2017, 9, 626. [CrossRef]

(C) 2018 by the authors. Licensee MDPI, Basel, Switzerland. This article is an open access article distributed under the terms and conditions of the Creative Commons Attribution (CC BY) license (http://creativecommons.org/licenses/by/4.0/). 